\title{
Chemical Yields from Supernovae and Hypernovae
}

\author{
Ken'ichi Nomoto ${ }^{1,2}$, Shinya Wanajo ${ }^{1,2}$, Yasuomi Kamiya ${ }^{1,2}$, \\ Nozomu Tominaga ${ }^{3}$, and Hideyuki Umeda ${ }^{2}$ \\ ${ }^{1}$ Institute for the Physics and Mathematics of the Universe, University of Tokyo, Kashiwa, \\ Chiba 277-85668, Japan \\ email: nomoto@astron.s.u-tokyo.ac.jp \\ ${ }^{2}$ Department of Astronomy, University of Tokyo, Bunkyo-ku, Tokyo 113-0033, Japan \\ ${ }^{3}$ National Astronomical Observatory, Mitaka, Tokyo 113-0033, Japan
}

\begin{abstract}
We review the final stages of stellar evolution, supernova properties, and chemical yields as a function of the progenitor's mass. (1) 8 - $10 M_{\odot}$ stars are super-AGB stars when the $\mathrm{O}+\mathrm{Ne}+\mathrm{Mg}$ core collapses due to electron capture. These AGB-supernovae may constitute an SN 2008S-like sub-class of Type IIn supernovae. These stars produce little $\alpha$-elements and Fe-peak elements, but are important sources of Zn and light p-nuclei. (2) $10-90 M_{\odot}$ stars undergo Fe-core collapse. Nucleosynthesis in aspherical explosions is important, as it can well reproduce the abundance patterns observed in extremely metal-poor stars. (3) $90-140 M_{\odot}$ stars undergo pulsational nuclear instabilities at various nuclear burning stages, including $\mathrm{O}$ and Si-burning. (4) Very massive stars with $M \gtrsim 140 M_{\odot}$ either become pair-instability SNe, or undergo core-collapse to form intermediate mass black holes if the mass loss is small enough.
\end{abstract}

Keywords. Galaxy: halo — gamma rays: bursts - nuclear reactions, nucleosynthesis, abundances — stars: abundances — stars: AGB — supernovae: general

\section{Core-Collapse Supernovae and Progenitor Masses}

The final stages of massive star evolution, supernova properties, and their chemical yields depend on the progenitor's masses $M$ as follows (e.g., Arnett 1996):

(1) $8-10 M_{\odot}$ stars: These stars are on the AGB phase when the $\mathrm{O}+\mathrm{Ne}+\mathrm{Mg}$ core collapses due to electron captures. The exact mass range depends on the mass loss during the AGB phase. They undergo weak explosions being induced by neutrino heating. These stars produce little $\alpha$-elements and Fe-peak elements, but are important sources of Zn and light p-nuclei. These AGB supernovae may constitute an SN 2008S-like sub-class of Type IIn supernovae.

(2) 10 - $90 M_{\odot}$ stars: These stars undergo Fe-core collapse to form either a neutron star (NS) or a black hole $(\mathrm{BH})$, and produce a large amount of heavy elements from $\alpha$-elements and Fe-peak elements. Observations have shown that the explosions of these Fe-core collapse supernovae are quite aspherical. In the extreme case, the supernova energy is higher than $10^{52} \mathrm{erg} \mathrm{s}^{-1}$, i.e. a Hypernova. Nucleosynthesis in these jet-induced explosions is in good agreement with the abundance patterns observed in extremely metal-poor stars.

(3) 90 - $140 M_{\odot}$ stars: These massive stars undergo nuclear instabilities and associated pulsations ( $\epsilon$-mechanism) at various nuclear burning stages depending on the mass loss and thus metallicity. In particular, if the mass loss is negligible, pulsations of O-cores and/or Si-cores due to O, Si-burning could produce dynamical mass ejection. Eventually, these stars undergo Fe-core collapse. 


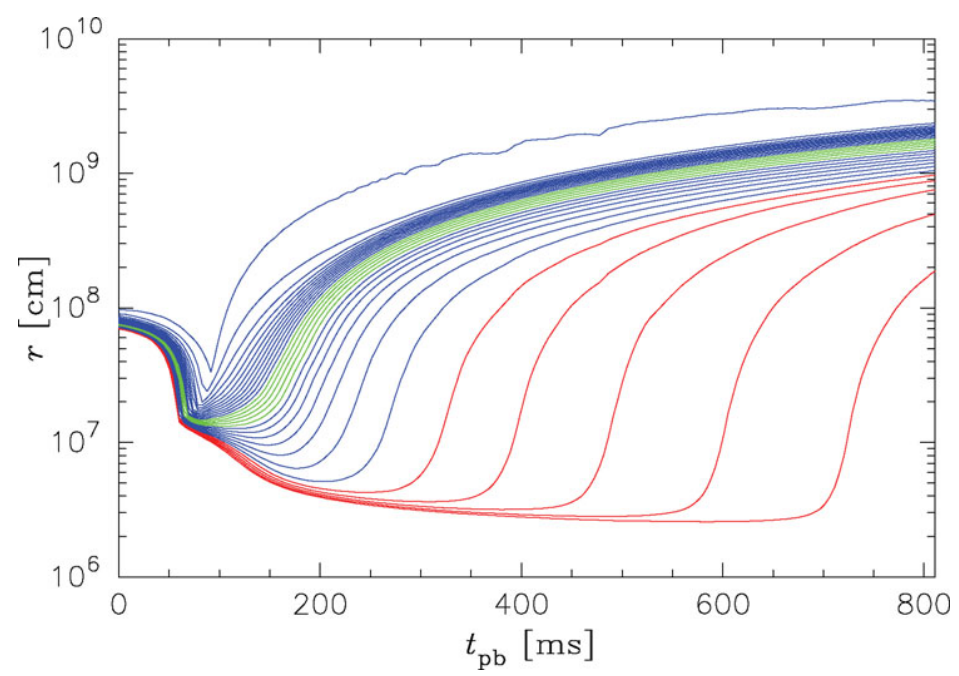

Figure 1. The change in the radius as a function of post-bounce time for material ejected from the collapsing O-Ne-Mg core (Kitaura \& Janka 2006).

(4) 140 - $300 M_{\odot}$ stars: If these very massive stars (VMS) do not lose much mass, they become pair-instability supernovae (PISN). The star is completely disrupted without forming a $\mathrm{BH}$ and thus ejects a large amount of heavy elements, especially Fe.

(5) Stars with $M \gtrsim 300 M_{\odot}$ : These VMSs are too massive to be disrupted by PISN but undergo core collapse (CVMS), forming an intermediate-mass black hole (IMBH). Some mass ejection could be possible, associated with the possible jet-induced explosion.

Here we summarize the properties of the above supernovae and chemical yields in some detail.

\section{8 - $10 M_{\odot}$ AGB Stars undergoing Electron Capture Supernovae}

\subsection{Collapse of $\mathrm{O}+\mathrm{Ne}+\mathrm{Mg}$ Core induced by Electron Capture}

For 8 - $10 M_{\odot}$ stars, electrons become degenerate already in a $\mathrm{C}+\mathrm{O}$ core. In a semidegenerate $\mathrm{C}+\mathrm{O}$ core, neutrino cooling leads to off-center ignition of carbon when the $\mathrm{C}+\mathrm{O}$ core mass exceeds the critical mass of $1.06 M_{\odot}$. The off-center carbon burning shell moves inward all the way to the center due to heat conduction (Nomoto 1984; Timmes \& Woosley 1992; Garcia-Berro et al. 1997).

After exhaustion of carbon in the central region, an $\mathrm{O}+\mathrm{Ne}+\mathrm{Mg}$ core forms. The core mass does not exceed the critical mass of $1.37 M_{\odot}$ for neon ignition and, hence, neon burning is never ignited (Nomoto 1984). Then the $\mathrm{O}+\mathrm{Ne}+\mathrm{Mg}$ core becomes strongly degenerate. The envelope becomes similar to the asymptotic giant-branch (super-AGB) stars (Hashimoto, Iwamoto, \& Nomoto 1993; Poelarends et al. 2008) with a thin He burning shell that undergoes thermal pulses and $s$-process nucleosynthesis.

The final fate depends on the competition between the mass loss that reduces the envelope mass and the increase in the core mass through the $\mathrm{H}$-He shell burning. If the mass loss is fast, an $\mathrm{O}+\mathrm{Ne}+\mathrm{Mg}$ white dwarf is formed, which could be the case for $8 M_{\odot}$ - $\mathrm{M}_{\text {up }}$ stars, where $\mathrm{M}_{\mathrm{up}} \sim 9 \pm 0.5 M_{\odot}$ being smaller for smaller metallicity (Poelarends et al. 2008). For $\mathrm{M}_{\mathrm{up}}-10 M_{\odot}$ stars, the core mass grows to $1.38 M_{\odot}$ and the central density reaches $4 \times 10^{9} \mathrm{~g} \mathrm{~cm}^{-3}$. The electron Fermi energy exceeds the threshold for electron captures ${ }^{24} \mathrm{Mg}\left(\mathrm{e}^{-}, \nu\right){ }^{24} \mathrm{Na}\left(\mathrm{e}^{-}, \nu\right){ }^{20} \mathrm{Ne}$ and ${ }^{20} \mathrm{Ne}\left(\mathrm{e}^{-}, \nu\right){ }^{20} \mathrm{~F}\left(\mathrm{e}^{-}, \nu\right){ }^{20} \mathrm{O}$. The resultant decrease in $Y_{e}$ triggers collapse (Nomoto 1987). 


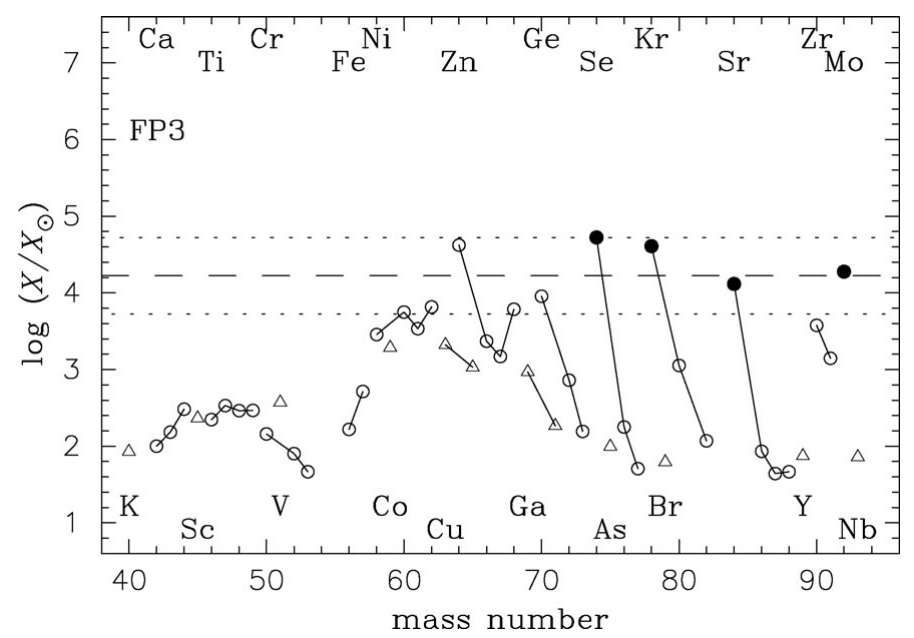

Figure 2. Mass fractions of isotopes (after decay) in the ejecta of model FP3 (Wanajo et al. 2008 ) relative to their solar values (Lodders 2003) as a function of the mass number. The even- $Z$ and odd- $Z$ isotopes are denoted by open circles and triangles, respectively. The $p$-nuclei are represented by filled symbols. The dotted horizontal lines indicate a "normalization band" between the largest production factor and a factor of ten smaller than that, along with the median value (dashed line).

The hydrodynamical behavior of collapse and bounce is somewhat different from the iron core collapse of more massive stars (Fig. 1: Kitaura \& Janka 2006). The explosion energy is as low as $E \sim 10^{50} \mathrm{erg}$. The explosion is also suggested be close to spherical, thus producing little pulsar kick. The existence of pulsars in globular clusters might be explained by the electron capture supernovae (Kalgera et al. 2008).

\subsection{Nucleosynthesis in Electron Capture Supernovae}

Nucleosynthesis in the supernova explosion of a $9 M_{\odot}$ star has been investigated (Hofman et al. 2008; Wanajo et al. 2008) using thermodynamic trajectories taken from the explosion model (Kitaura \& Janka 2006). Here we summarize the results by Wanajo et al. (2008).

1. The unmodified model produces small amounts of $\alpha$-elements and iron, but large amounts of ${ }^{64} \mathrm{Zn},{ }^{70} \mathrm{Ge}$, and in particular, ${ }^{90} \mathrm{Zr}$, with some light $p$-nuclei (e.g., ${ }^{92} \mathrm{Mo}$; Fig. 2). This is due to the ejection of a large amount of neutron-rich matter $\left(Y_{e}=\right.$ $0.46-0.49)$, and might put severe constraints on the frequency of occurrence of this type of supernovae (Hofman et al. 2008). However, the production of ${ }^{90} \mathrm{Zr}$ does not serve as a strong constraint, because it is easily affected by a small variation of $Y_{e}$ (see below).

2. The overproduction of ${ }^{90} \mathrm{Zr}$ becomes more moderate if the minimum $Y_{e}$ is only $1-2 \%$ larger than that in the unmodified model. Such a change in the initial $Y_{e}$ profile might be caused by convection that is not considered in the 1-D simulation (Kitaura \& Janka 2006). In this case (model FP3: Fig. 2), the largest overproduction, which is shared by ${ }^{64} \mathrm{Zn},{ }^{70} \mathrm{Se}$, and ${ }^{78} \mathrm{Kr}$, falls to one-tenth that of the unmodified model. The ${ }^{64} \mathrm{Zn}$ production provides an upper limit to the occurrence of exploding O-Ne-Mg cores at about $20 \%$ of all core-collapse supernovae.

3. The ejecta mass of ${ }^{56} \mathrm{Ni}$ is $0.002-0.004 M_{\odot}$, much smaller than the $\sim 0.1 M_{\odot}$ in more massive progenitors. Convective motions near the mass cut may also affect the $Y_{e}$-distribution and thus the ${ }^{56} \mathrm{Ni}$ mass. See Wanajo et al. (2008) for a recent comparison between the electron capture supernova yields and abundances in the Crab Nebula. 

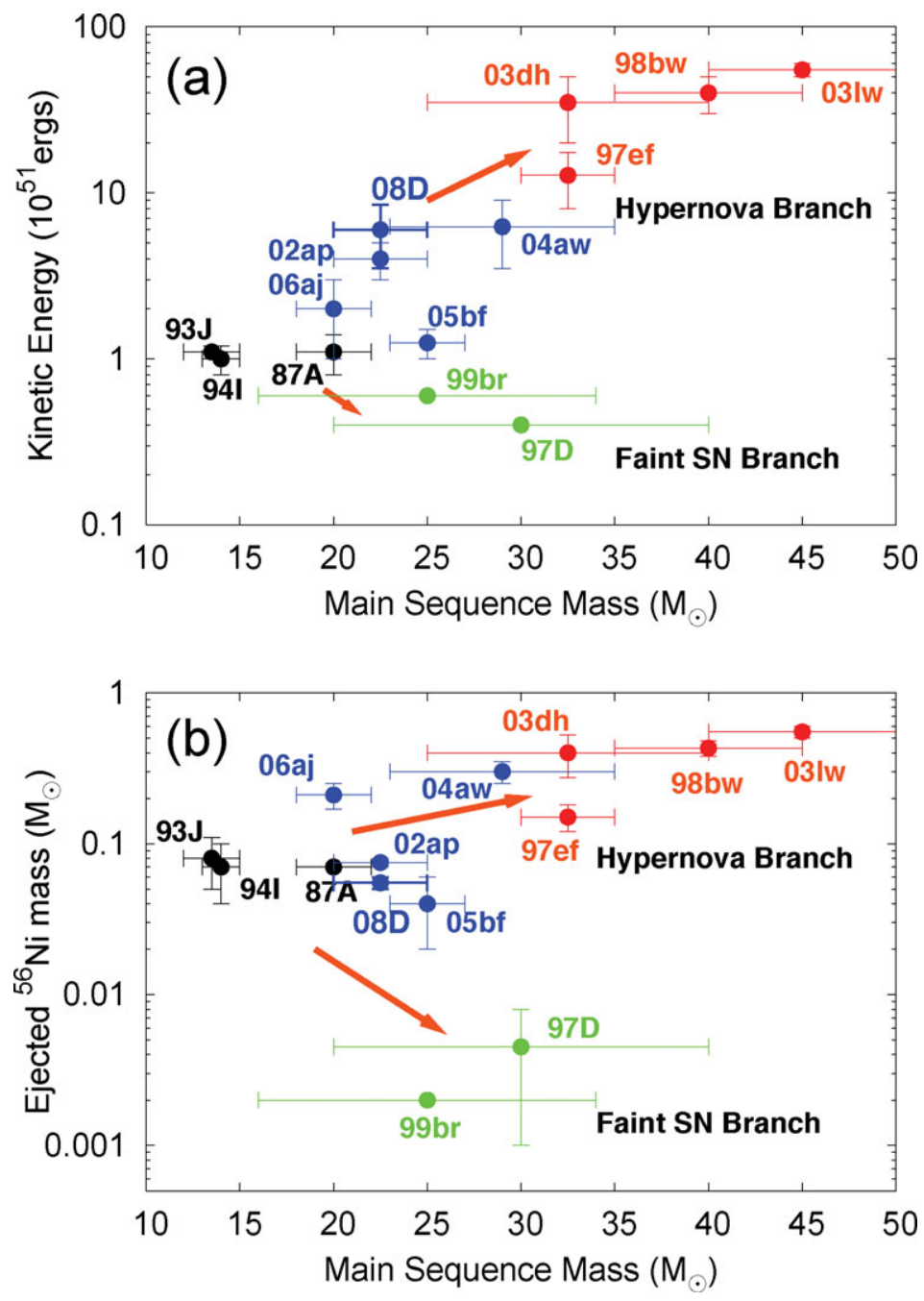

Figure 3. Upper: The kinetic explosion energy $E$ as a function of the main sequence mass $M$ of the progenitors for several supernovae/hypernovae. Hypernovae are the SNe with $E_{51}>10$. Lower: Same as the upper panel, but for the ejected mass of ${ }^{56} \mathrm{Ni}$.

\subsection{Connection to Faint Supernovae}

The expected small amount of ${ }^{56} \mathrm{Ni}$ as well as the low explosion energy of electron capture supernovae have been proposed as an explanation of the observed properties of lowluminosity SNe IIP, such as SN 1997D (Chugai \& Utrobin 2000; Kitaura \& Janka 2006) and of the low luminosity of SN 2008S-like transients (Prieto et al. 2008; Thompson et al. 2008). The estimated ${ }^{56} \mathrm{Ni}$ masses of $\sim 0.002-0.008 M_{\odot}$ for observed low-luminosity SNe II-P (Zampieri et al. 2003; Hendry et al. 2005) are in reasonable agreement with the presented results from $\mathrm{O}-\mathrm{Ne}-\mathrm{Mg}$ core explosions. An alternative possibility of such supernovae is that more massive stars $\left(\gtrsim 20 M_{\odot}\right)$ with low explosion energies suffer from fallback of freshly synthesized ${ }^{56} \mathrm{Ni}$ (Turatto et al. 1998; Nomoto et al. 2003; Zampieri et al. 2003). A recent analysis of the progenitors of SNe IIP by Smartt et al. (2008) favors low mass progenitors. A lack of $\alpha$-elements such as $\mathrm{O}$ and $\mathrm{Mg}$ in the case of collapsing $\mathrm{O}-\mathrm{Ne}-\mathrm{Mg}$ cores will be a key to spectroscopically distinguish between these two scenarios. 

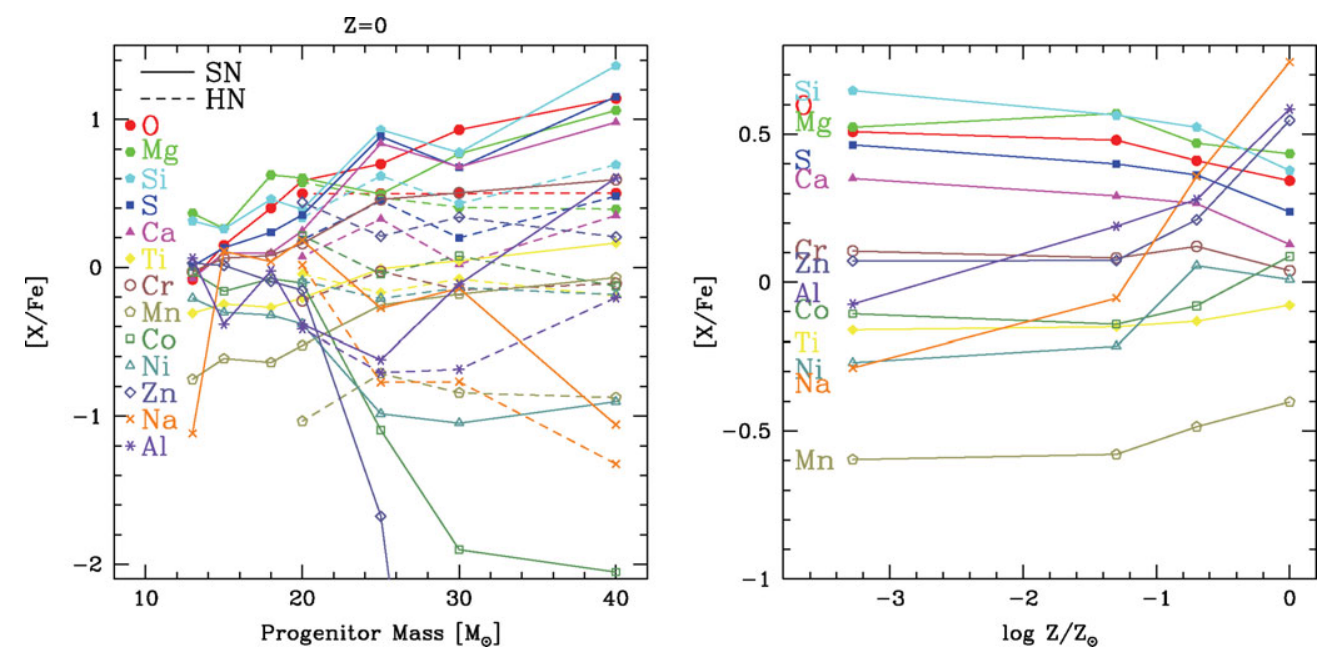

Figure 4. (Left:) Relative abundance ratios as a function of progenitor mass with $Z=0$. The solid and dashed lines show normal SNe II with $E_{51}=1$ and HNe. (Right:) The IMF weighted abundance ratios as a function of metallicity of progenitors, where the $\mathrm{HN}$ fraction $\epsilon_{\mathrm{HN}}=0.5$ is adopted. Results for $Z=0$ are plotted at $\log Z / Z_{\odot}=-4$ (Nomoto et al. 2006; Kobayashi et al. 2006).

Recently, the progenitor of SN 2008S was discovered in the infrared, and it has been suggested that it was an AGB star (Prieto et al. 2008). If so, SN 2008S could belong to the electron capture supernovae.

The envelope of the AGB star is carbon-enhanced (Nomoto 1987). Then dust could easily be formed to induce mass loss. This may result in a deeply dust-enshrouded object such as the progenitor of SN 2008S (Prieto et al. 2008; Thompson et al. 2008). This might also imply that the mass range of the stars that end their lives as O-Ne-Mg supernovae is $\sim 9.5-10 M_{\odot}$; their frequency, $\sim 7-8 \%$ of all the core-collapse events, satisfies the constraint from our nucleosynthesis results $(<30 \%)$.

\section{10 - $90 M_{\odot}$ Stars undergoing Aspherical Explosions}

These stars undergo Fe-core collapse and become Type II-P (plateau) supernovae (SNe II-P) if the red-supergiant-size H-rich envelope remains, and Type Ibc supernovae if the H-rich envelope has been stripped off by a stellar wind or Roche-lobe overflow. These $\mathrm{SNe}$ are the major sources of heavy elements from $\mathrm{C}$ to the Fe-peak.

Their yields depend on the progenitor's mass $M$, metallicity, and the explosion energy $E$. From the comparison between the observed and calculated spectra and light curves of supernovae, we can estimate $M$ and $E$ as shown in Figure 3 (Nomoto et al. 2006).

Three SNe (SNe 1998bw, 2003dh, and 2003lw) are associated with long Gamma-Ray Bursts (GRBs) (e.g., Woosley \& Bloom 2006). The progenitors of these GRB-SNe tend to be more massive than $\sim 30 M_{\odot}$. Also GRB-SNe are all very energetic with the kinetic energy $E$ exceeding $10^{52} \mathrm{erg}$, more than 10 times the kinetic energy of normal corecollapse SNe. Here we use the term 'Hypernova (HN)' to describe such hyper-energetic supernovae with $E_{51}=E / 10^{51}$ erg $\gtrsim 10$ (Fig.3; Nomoto et al. 2004, 2006; Kobayashi et al. 2006). 

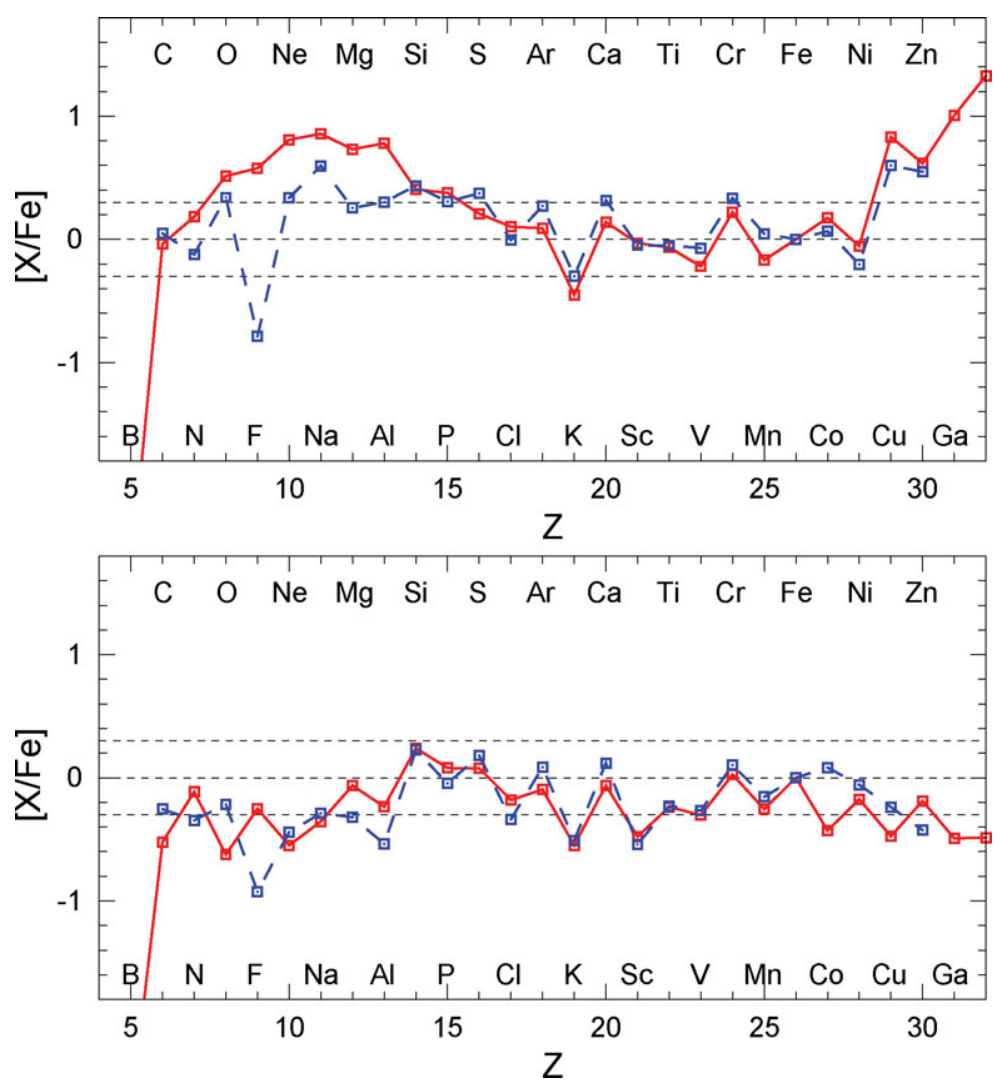

Figure 5. (Upper): Comparison of yields for $M=15 M_{\odot}, Z=0.02$, and $E=1 \times 10^{51} \mathrm{erg}$ (red-solid: Nomoto et al. 2006; blue-dashed: Limongi et al. 2000). (Lower): Same as upper, but for $M=25 M_{\odot}$.

\subsection{Supernova and Hypernova Yields}

Theoretical models of stellar evolution depend on the treatment of complicated physical processes, such as mixing due to convection and rotation, convective overshooting, mass loss, etc. Thus SN yields obtained by various groups are not necessarily in agreement. Figure 5 compares the yields of the models with $E=1 \times 10^{51} \mathrm{erg}$ and $Z=0.02$ for $M=15 M_{\odot}$ (upper) and $M=25 M_{\odot}$ (lower) (Nomoto et al. 2006; Limongi et al. 2000). These models include mass loss but not rotation. It is seen that two yields are in good agreement.

Figure 6 (upper) compares the yields of models with $E=1 \times 10^{51} \mathrm{erg}$ and $Z=0.00$ for $M=20 M_{\odot}$ between the three groups (Nomoto et al. 2006; Limongi et al. 2000; Heger \& Woosley 2008). These three yields are in good agreement. The smooth pattern in Heger \& Woosley (2008) is due to the mixing-fallback effect (Umeda \& Nomoto 2002) being taken into account in their model. This implies that the difference in the treatment of such mixing-fallback during the explosion is larger than other differences in presupernova models among the three groups.

Figure 6 (upper) also compares the observed averaged abundance pattern of the EMP stars (Cayrel et al. 2004) with the three theoretical models. We note that theoretical predictions of $\mathrm{Zn}, \mathrm{Co}, \mathrm{Ti} / \mathrm{Fe}$ are much smaller than the observed ratios. The underproduction of these elements relative to $\mathrm{Fe}$ is much improved in the hypernova models 

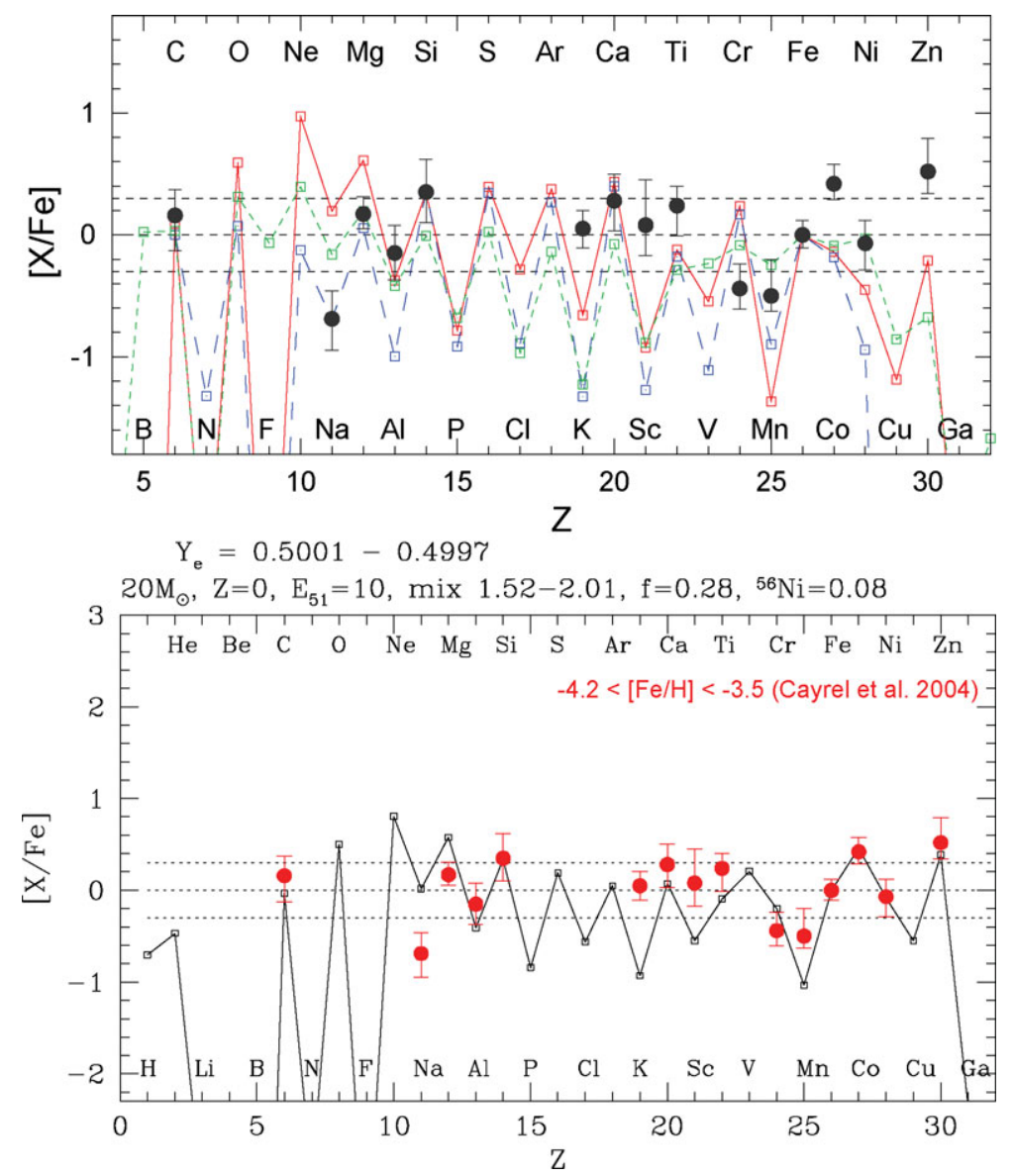

Figure 6. Averaged elemental abundances of stars with $[\mathrm{Fe} / \mathrm{H}]=-3.7$ (Cayrel et al. 2004) compared with yields for $M=20 M_{\odot}$ and $Z=0.0$ (upper: Nomoto et al. 2006; Limongi et al. 2000; Heger \& Woosley 2008) and the hypernova yield (lower: $20 M_{\odot}, E_{51}=10$ ).

(lower); this suggests that hypernovae play an important role in the chemical enrichment during early galactic evolution.

In the following section, therefore, we focus on nucleosynthesis in the high energy jet-induced explosions (Tominaga et al. 2007; Tominaga 2008). In the jet-like explosion, fallback can occur even for $E>10^{52} \mathrm{erg}$.

\subsection{Nucleosynthesis in Jet-Induced Explosions and GRB-SN Connection}

The observed late-time spectra indicate that the explosions of these Fe-core collapse supernovae are quite aspherical (Maeda et al. 2008; Modjaz et al. 2008). The extreme case is the hyper-aspherical explosions induced by relativistic jet(s) as seen in the GRBSNe.

Recent studies of nucleosynthesis in jet-induced explosions have revealed the connection between GRBs and EMP stars as summarized in Figure 7 (Tominaga et al. 2007; Tominaga 2008). In this model for the $40 M_{\odot}$ star, the jets are injected at a radius $R_{0} \sim 900$ $\mathrm{km}$ with energy deposition rates in the range $\dot{E}_{\mathrm{dep}, 51} \equiv \dot{E}_{\mathrm{dep}} / 10^{51} \mathrm{ergs} \mathrm{s}^{-1}=0.3-1500$. The diversity of $\dot{E}_{\text {dep }}$ is consistent with the wide range of the observed isotropic equivalent $\gamma$-ray energies and timescales of GRBs (Amati et al. 2007 and references therein). 


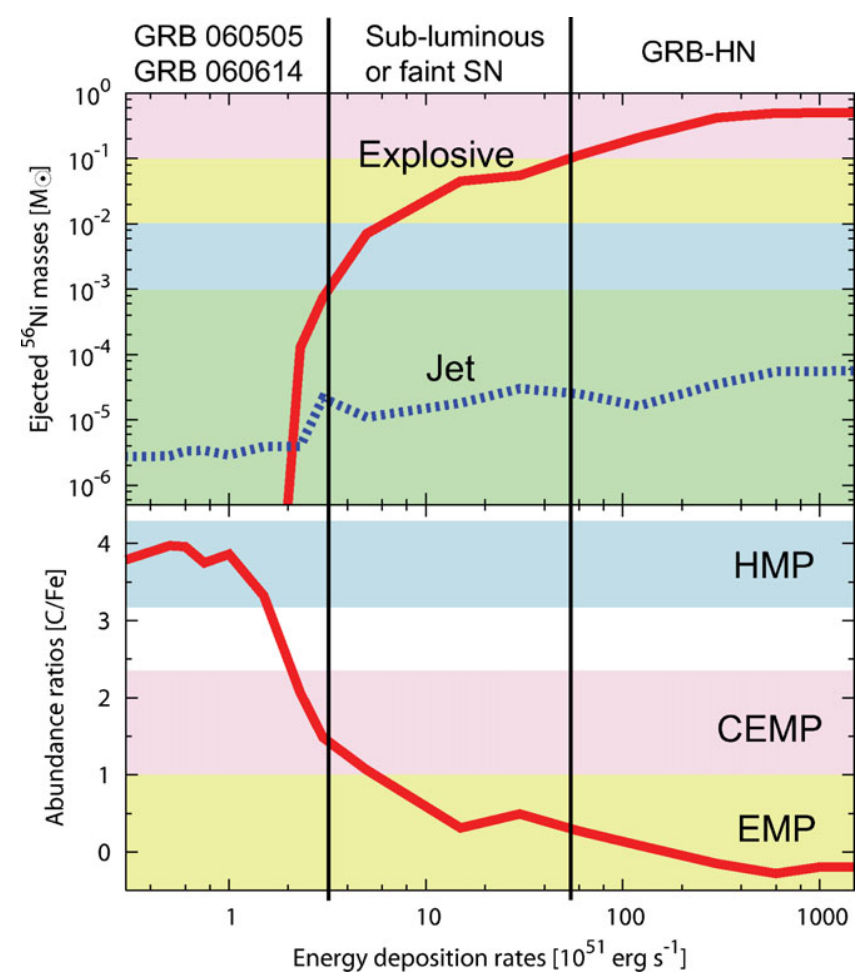

Figure 7. Upper: The ejected ${ }^{56} \mathrm{Ni}$ mass (red: explosive nucleosynthesis products, blue: the jet contribution) as a function of the energy deposition rate (Tominaga et al. 2007). The background color shows the corresponding SNe (red: GRB-HNe, yellow: sub-luminous SNe, blue: faint SNe, green: GRBs 060505 and 060614). Vertical lines divide the resulting SNe according to their brightness. Lower: the dependence of abundance ratio $[\mathrm{C} / \mathrm{Fe}]$ on the energy deposition rate. The background color shows the corresponding metal-poor stars (yellow: EMP, red: CEMP, blue: HMP stars).

Variations of activities of the central engines, possibly corresponding to different rotational velocities or magnetic fields, may well produce the variation of $\dot{E}_{\mathrm{dep}}$.

The ejected $M\left({ }^{56} \mathrm{Ni}\right)$ depends on $\dot{E}_{\text {dep }}$ as follows (Fig. 7). Generally, higher $\dot{E}_{\text {dep }}$ leads to the synthesis of larger $M\left({ }^{56} \mathrm{Ni}\right)$ in explosive nucleosynthesis because of higher postshock densities and temperatures (e.g., Maeda \& Nomoto 2003; Nagataki et al. 2006). If $\dot{E}_{\mathrm{dep}, 51} \gtrsim 60$, we obtain $M\left({ }^{56} \mathrm{Ni}\right) \gtrsim 0.1 M_{\odot}$, which is consistent with the brightness of GRB-HNe. Some $\mathrm{C}+\mathrm{O}$ core material is ejected along the jet direction, but a large amount of material along the equatorial plane falls back.

For $\dot{E}_{\mathrm{dep}, 51} \gtrsim 60$, the remnant mass is initially $M_{\mathrm{rem}}^{\text {start }} \sim 1.5 M_{\odot}$ and grows as materials is accreted from the equatorial plane. The final $\mathrm{BH}$ mass is generally larger for smaller $\dot{E}_{\text {dep }}$. The final BH masses range from $M_{\mathrm{BH}}=10.8 M_{\odot}$ for $\dot{E}_{\mathrm{dep}, 51}=60$ to $M_{\mathrm{BH}}=5.5 M_{\odot}$ for $\dot{E}_{\mathrm{dep}, 51}=1500$, which are consistent with the observed masses of stellar-mass BHs (Bailyn et al. 1998). The model with $\dot{E}_{\text {dep }, 51}=300$ synthesizes $M\left({ }^{56} \mathrm{Ni}\right) \sim 0.4 M_{\odot}$, and the final mass of $\mathrm{BH}$ left after the explosion is $M_{\mathrm{BH}}=6.4 M_{\odot}$.

For low energy deposition rates $\left(\dot{E}_{\mathrm{dep}, 51}<3\right)$, in contrast, the ejected ${ }^{56} \mathrm{Ni}$ masses $\left(M\left({ }^{56} \mathrm{Ni}\right)<10^{-3} M_{\odot}\right)$ are smaller than the upper limits for GRBs 060505 and 060614 (Della Valle et al. 2006; Fynbo et al. 2006; Gal-Yam et al. 2006).

If the explosion is viewed from the jet direction, we would observe the GRB without SN re-brightening. This may be the situation for GRBs 060505 and 060614 . In particular, 


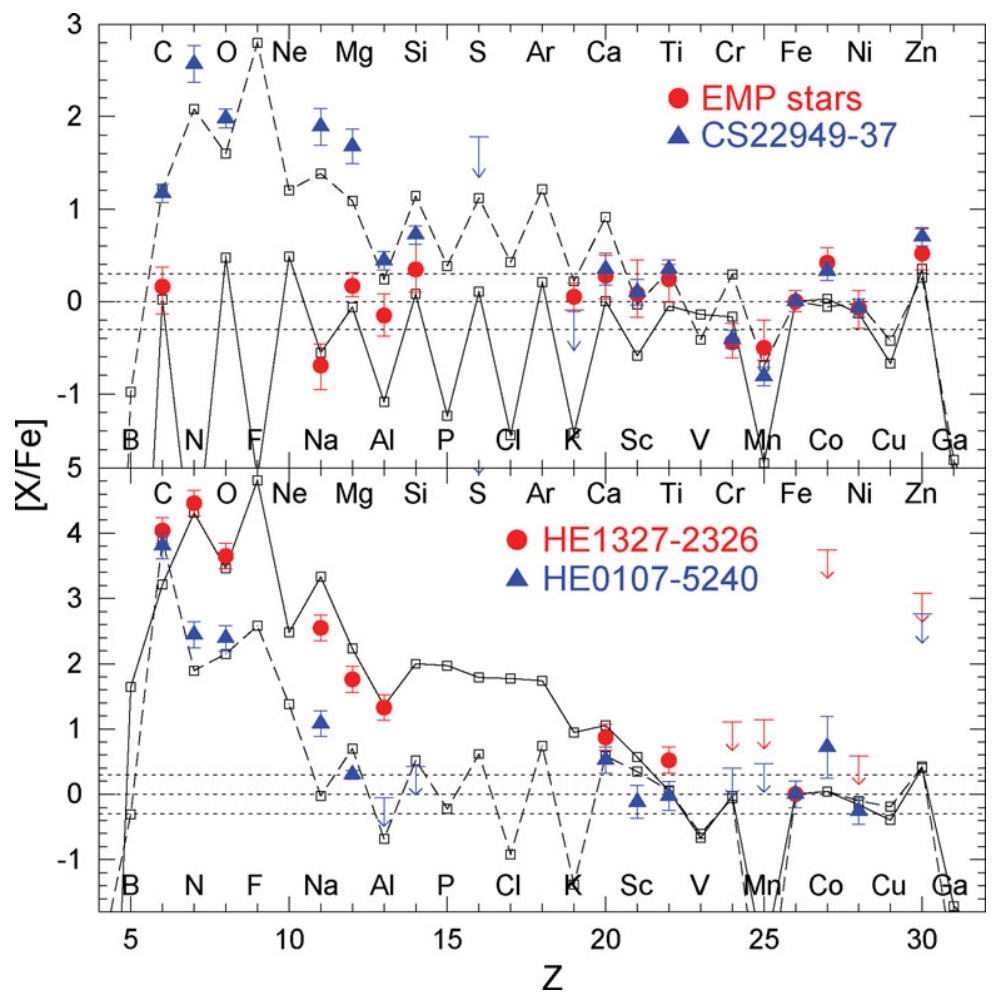

Figure 8. A comparison of the abundance patterns between metal-poor stars and models (Tominaga et al. 2007). Upper: typical EMP stars (red dots, Cayrel et al. 2004) and CEMP (blue triangles, CS 22949-37, Depagne et al. 2002) and models with $\dot{E}_{\mathrm{dep}, 51}=120$ (solid line) and $=3.0$ (dashed line). Lower: HMP stars: HE 1327-2326, (red dots, e.g., Frebel et al. 2005), and HE 0107-5240, (blue triangles, Christlieb et al. 2002, Bessell \& Christlieb 2005) and models with $\dot{E}_{\mathrm{dep}, 51}=1.5$ (solid line) and $=0.5$ (dashed line).

for $\dot{E}_{\text {dep }, 51}<1.5,{ }^{56} \mathrm{Ni}$ cannot be synthesized explosively, and the jet component of the Fe-peak elements dominates the total yields (Fig. 8). The models eject very little $M\left({ }^{56} \mathrm{Ni}\right)$ $\left(\sim 10^{-6} M_{\odot}\right)$.

For intermediate energy deposition rates $\left(3 \lesssim \dot{E}_{\mathrm{dep}, 51}<60\right)$, the explosions eject $10^{-3} M_{\odot} \lesssim M\left({ }^{56} \mathrm{Ni}\right)<0.1 M_{\odot}$, and the final $\mathrm{BH}$ masses are $10.8 M_{\odot} \lesssim M_{\mathrm{BH}}<15.1 M_{\odot}$. The resulting $\mathrm{SN}$ is faint $\left(M\left({ }^{56} \mathrm{Ni}\right)<0.01 M_{\odot}\right)$ or sub-luminous $\left(0.01 M_{\odot} \lesssim M\left({ }^{56} \mathrm{Ni}\right)<\right.$ $\left.0.1 M_{\odot}\right)$.

\subsection{Abundance Patterns of Extremely Metal-Poor Stars}

The abundance ratio $[\mathrm{C} / \mathrm{Fe}]$ depends on $\dot{E}_{\text {dep }}$ as follows. Lower $\dot{E}_{\text {dep }}$ yields larger $M_{\mathrm{BH}}$ and thus larger $[\mathrm{C} / \mathrm{Fe}]$, because the infall reduces the amount of inner core material $(\mathrm{Fe})$ relative to that of outer material (C) (see also Maeda \& Nomoto 2003). As in the case of $M\left({ }^{56} \mathrm{Ni}\right),[\mathrm{C} / \mathrm{Fe}]$ changes dramatically at $\dot{E}_{\mathrm{dep}, 51} \sim 3$.

The observed abundance patterns of EMP stars are good indicators of SN nucleosynthesis because the Galaxy was effectively unmixed at $[\mathrm{Fe} / \mathrm{H}]<-3$ (e.g., Tumlinson 2006). They are classified into three groups according to $[\mathrm{C} / \mathrm{Fe}]$ :

(1) $[\mathrm{C} / \mathrm{Fe}] \sim 0$, normal EMP stars $(-4<[\mathrm{Fe} / \mathrm{H}]<-3$, e.g., Cayrel et al. 2004);

(2) $[\mathrm{C} / \mathrm{Fe}] \gtrsim+1$, Carbon-enhanced EMP (CEMP) stars $(-4<[\mathrm{Fe} / \mathrm{H}]<-3$, e.g., CS 22949-37, Depagne et al. 2002); 
Table 1. Stability of Pop III and Pop I massive stars: $\bigcirc$ and $\times$ indicate that the star is stable or unstable, respectively. The $e$-folding time for the fundamental mode is shown after $\times$ in units of $10^{4} \mathrm{yr}$ (Nomoto et al. 2003).

\begin{tabular}{ccccccc}
\hline$M\left(M_{\odot}\right)$ & 80 & 100 & 120 & 150 & 180 & 300 \\
\hline Pop III & $\bigcirc$ & $\bigcirc$ & $\bigcirc$ & $\times(9.03)$ & $\times(4.83)$ & $\times(2.15)$ \\
Pop I & $\bigcirc$ & $\times(7.02)$ & $\times(2.35)$ & $\times(1.43)$ & $\times(1.21)$ & $\times(1.71)$ \\
\hline
\end{tabular}

(3) $[\mathrm{C} / \mathrm{Fe}] \sim+4$, hyper metal-poor $(\mathrm{HMP})$ stars $([\mathrm{Fe} / \mathrm{H}]<-5$, e.g., HE 0107-5240, Christlieb et al. 2002; Bessell \& Christlieb 2005; HE 1327-2326, Frebel et al. 2005).

Figure 8 shows that the abundance patterns of the averaged normal EMP stars, the CEMP star CS 22949-37, and the two HMP stars (HE 0107-5240 and HE 1327-2326) are well reproduced by the models with $\dot{E}_{\mathrm{dep}, 51}=120,3.0,1.5$, and 0.5 , respectively. The model for the normal EMP stars ejects $M\left({ }^{56} \mathrm{Ni}\right) \sim 0.2 M_{\odot}$, i.e., a factor of 2 less than SN 1998bw. On the other hand, the models for the CEMP and the HMP stars eject $M\left({ }^{56} \mathrm{Ni}\right) \sim 8 \times 10^{-4} M_{\odot}$ and $4 \times 10^{-6} M_{\odot}$, respectively, which are always smaller than the upper limits for GRBs 060505 and 060614. The N/C ratio in the models for CS 22949-37 and $\mathrm{HE} 1327-2326$ is enhanced by partial mixing between the He and $\mathrm{H}$ layers during presupernova evolution (Iwamoto et al. 2005).

To summarize, (1) the explosions with large energy deposition rate, $\dot{E}_{\mathrm{dep}}$, are observed as GRB-HNe, and their yields can explain the abundances of normal EMP stars, and (2) the explosions with small $\dot{E}_{\text {dep }}$ are observed as GRBs without bright SNe and can be responsible for the formation of the CEMP and the HMP stars. We thus propose that GRB-HNe and GRBs without bright SNe belong to a continuous series of BH-forming massive stellar deaths with relativistic jets of different $\dot{E}_{\mathrm{dep}}$.

\section{4. $90-140 M_{\odot}$ Stars undergoing Pulsational Nuclear Instabilites}

These massive stars undergo nuclear instabilities and associated pulsations ( $\epsilon$-mechanism) at various nuclear burning stages. Because of the large contribution of radiation pressure in these stars, dynamical stability is very close to neutral. Even a slight contribution of electron-positron pair creation affects the stability. Thus the pulsation behavior is sensitive to their mass, and thus to the mass loss rate and metallicity.

To determine the above upper mass limit, the non-adiabatic stability of massive Pop III $(Z=0)$ stars has been analyzed (Ibrahim et al. 1981; Baraffe et al. 2001; Nomoto et al. 2003). As summarized in Table 1 (Nomoto et al. 2003), the critical mass of a Pop III star is $128 M_{\odot}$, while that of a Pop I star is $94 M_{\odot}$. This difference comes from the very compact structure (with high central temperature) of Pop III stars. Stars more massive than the critical mass will undergo pulsation and mass loss. We note that the $e$-folding time of instability is much longer for Pop III stars than Pop I stars with the same mass, and thus the mass loss rate is much lower. Thus, massive Pop III stars could survive the instabilities without losing much mass.

Massive Pop III stars are formed through mass accretion, starting from a tiny core through collapse (e.g., Yoshida et al. 2008). Such an evolution with mass accretion starting from $M \sim 1 M_{\odot}$ has recently been studied by Ohkubo et al. $(2006,2008)$.

Figure 9 shows the evolutionary tracks of the central density and temperature in the later phases. For the models with mass accretion (M-2, YII), the central entropy in the early stage is low, corresponding to the small stellar mass. During the main-sequence 


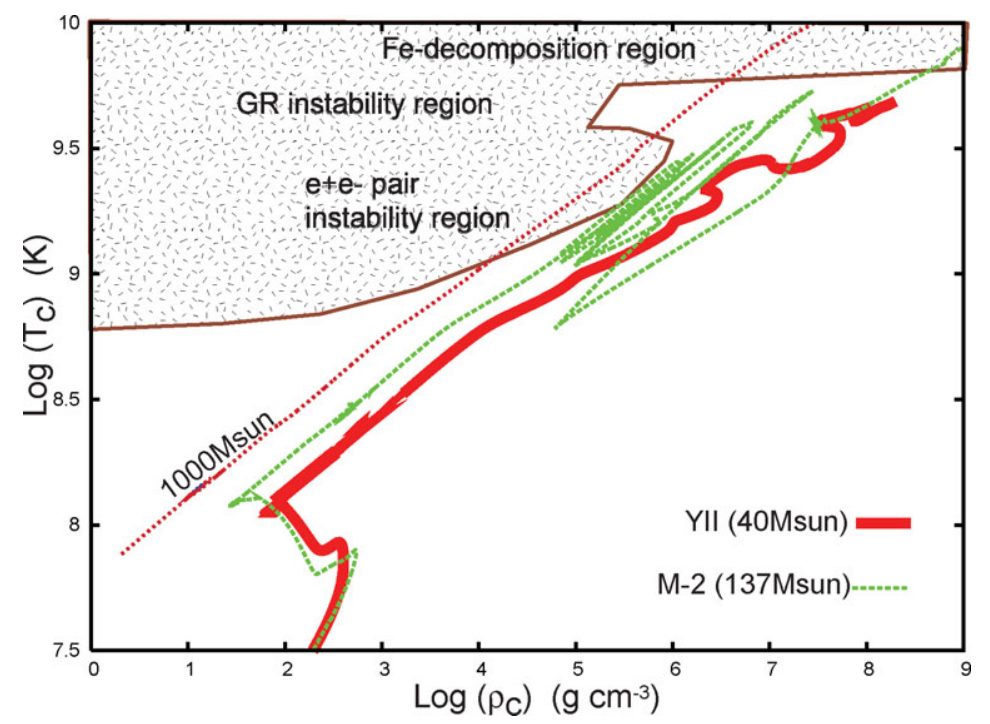

Figure 9. Evolutionary tracks of the central temperature and central density of very massive stars (Ohkubo et al. 2008). The numbers in brackets are the final masses for models YII and M-2. The $1000 M_{\odot}$ stars (Ohkubo et al. 2006) are also shown.

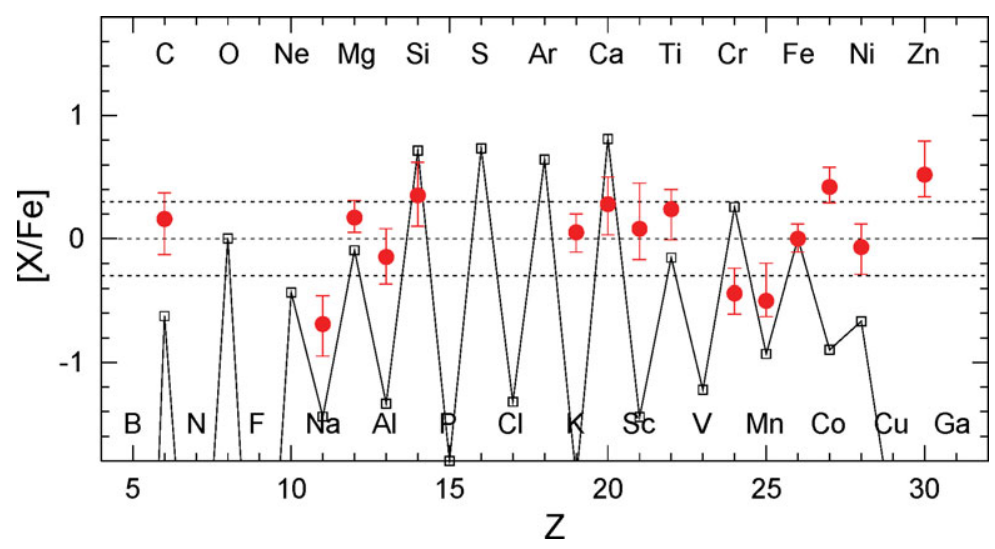

Figure 10. Comparison of the abundance patterns in the pair-instability supernova model $\left(M=200 M_{\odot}\right.$; Umeda \& Nomoto 2002) and extremely metal-poor stars (Cayrel et al. 2004).

phase, the stellar mass increases to $\sim 40 M_{\odot}(\mathrm{YII})$ and $\sim 137 M_{\odot}(\mathrm{M}-2)$, and the densitytemperature track shifts toward corresponding higher entropy through hydrogen burning.

The star YII $\left(\sim 40 M_{\odot}\right)$ ends its life in Fe-core collapse to form a black hole. The star M-2, whose final mass is $137 M_{\odot}$, undergoes nuclear instability due to oxygen and silicon burning and pulsates, as seen in Figure 9. Such pulsations are seen in stars with masses $90 M_{\odot} \lesssim M \lesssim 140 M_{\odot}$ (Nomoto et al. 2005; Woosley et al. 2007; Umeda \& Nomoto 2008; Ohkubo et al. 2008). In the extreme case, the pulsation could induce dynamical mass ejection and optical brightening as might be observed in the brightest SN 2006gy (Woosley et al. 2007). The nuclear energy released is not sufficient to explode the whole star. After several oscillations, the star finally collapses to form a black hole. 


\section{140 - $300 M_{\odot}$ Stars undergoing Pair-Instability Supernovae}

These very massive stars (VMS) undergo pair-creation instability and are disrupted completely by explosive oxygen burning, as pair-instability supernovae (PISNe) (e.g., Arnett 1996; Umeda \& Nomoto 2002; Heger \& Woosley 2002).

The abundance patterns of the ejected material for the $200 M_{\odot}$ star (Umeda \& Nomoto 2002) are compared with EMP stars (Cayrel et al. 2004) in Figure 10. It is clear that PISN ejecta cannot be consistent with the large C/Fe observed in HMP stars and other C-rich EMP stars. Also, the abundance ratios of iron-peak elements $([\mathrm{Zn} / \mathrm{Fe}]<-0.8$ and $[\mathrm{Co} / \mathrm{Fe}]<-0.2)$ in the PISN ejecta cannot explain the large $\mathrm{Zn} / \mathrm{Fe}$ and $\mathrm{Co} / \mathrm{Fe}$ ratios in typical EMP stars.

Therefore, the supernova progenitors that are responsible for the formation of EMP stars are most likely in the range of $M \sim 20-140 M_{\odot}$, but not more massive than 140 $M_{\odot}$. The absence of any indication of PISNe in EMP stars might imply that 140 - 300 $M_{\odot}$ stars undergo significant mass loss, thus evolving into Fe core-collapse.

\section{Very Massive Stars with $M>300 M_{\odot}$ and Intermediate Mass Black Holes}

It is possible that the First Stars were even more massive than $\sim 300 M_{\odot}$ (Ohkubo et al. 2006, 2008) if rapid mass accretion continues during the whole main-sequence phase of Pop III stars. [Another possible scenario for any metallicity is that VMSs are formed by merging of less massive stars in the environment of very dense star clusters (e.g., Ebisuzaki et al. 2001; Portezies Zwart \& van den Heuvel 2007)].

Such massive stars undergo core-collapse (CVMS: core-collapse VMS) as seen from the $1000 M_{\odot}$ star track in Figure 9. If such stars formed rapidly rotating black holes, jet-like mass ejection could produce processed material (Ohkubo et al. 2006). In fact, for moderately aspherical explosions, the patterns of nucleosynthesis match the observational data of both intracluster medium and M82 (Ohkubo et al. 2006). This result suggests that explosions of CVMS contribute significantly to the chemical evolution of gases in clusters of galaxies. For Galactic halo stars, predicted $[\mathrm{O} / \mathrm{Fe}]$ ratios are smaller than the observational abundances. This result may support the view that Pop III CVMS could be responsible for the origin of intermediate mass black holes (IMBH).

\section{References}

Amati, L., Della Valle, M., Frontera, F., et al. 2007, A\&\&A 463, 913

Arnett, W. D. 1996, Supernovae and Nucleosynthesis (Princeton: Princeton Univ. Press)

Bailyn, C. D., Jain, R. K., Coppi, P., \& Orosz, J. A. 1998, ApJ 499, 367

Baraffe, I., Heger, A., \& Woosley, S. E. 2001, ApJ 550, 890

Bessell, M. S. \& Christlieb, N. 2005, in V. Hill et al. (eds.), From Lithium to Uranium, Proc. IAU Symposium No. 228 (Cambridge: Cambridge Univ. Press), 237

Cayrel, R., et al. 2004, A\&SA 416, 1117

Christlieb, N., et al. 2002, Nature 419, 904

Chugai, N. N. \& Utrobin, V.P. 2000, A\&A 354, 557

Della Valle, M., et al. 2006, Nature 444, 1050

Depagne, E., et al. 2002, A\& $A$ 390, 187

Ebisuzaki, T., et al. 2001, ApJ 562, L19

Fynbo, J. P. U., et al. 2006, Nature 444, 1047

Frebel, A., et al. 2005, Nature 434, 871

Garcia-Berro, E., Ritossa, C., \& Iben, I., Jr. 1997, ApJ 485, 765

Gal-Yam, A., et al. 2006, Nature 444, 1053 
Hashimoto, M., Iwamoto, K., \& Nomoto, K. 1993, ApJ 322, L206

Heger, A. \& Woosley, S. E. 2002, ApJ 567, 532

Heger, A. \& Woosley, S. E. 2008, arXiv:0803.3161

Hendry, M. A., et al. 2005, MNRAS 359, 906

Hofman et al. 2008, ApJ 395, L672

Ibrahim, A., Boury, A., \& Noels, A. 1981, A\& A 103, 390

Iwamoto, K., Mazzali, P. A., Nomoto, K., et al. 1998, Nature 395, 672

Iwamoto, N., Umeda, H., Tominaga, N., Nomoto, K., \& Maeda, K. 2005, Science 309, 451

Kalgero, J., et al. 2008, ApJ 670, 774

Kitaura \& Janka, T. 2006, A\&BA 133, 175

Kobayashi, C., Umeda, H., Nomoto, K., Tominaga, N., \& Ohkubo, T. 2006, ApJ 653, 1145

Limongi, M., Straniero, \& Chieffi, A. 2000, ApJS 129, 625

Lodders, K. 2003, ApJ 591, 1220

Maeda, K. \& Nomoto, K. 2003, ApJ 598, 1163

Maeda, K., et al. 2008, Science 319, 1220

Modjaz, M., et al. 2008, ApJ in press (arXiv:0801.0221)

Nagataki, S., Mizuta, A., \& Sato, K. 2006, ApJ 647, 1255

Nomoto, K. 1984, ApJ 277, 791

Nomoto, K. 1987, ApJ 322, 206

Nomoto, K., Maeda, K., Umeda, H., Ohkubo, T., Deng, J., \& Mazzali, P. 2003, in IAU Symp. 212, A Massive Star Odyssey, ed. V. D. Hucht, et al. (San Fransisco: ASP), 395

Nomoto, K., et al. 2004, in C. L. Fryer (ed.), Stellar Collapse (Astrophysics and Space Science: Kluwer), p. 277 (astro-ph/0308136)

Nomoto, K., et al. 2005, in The Fate of Most Massive Stars, ed. R. Humphreys \& K. Stanek (ASP Ser. 332), 374 (astro-ph/0506597)

Nomoto, K., et al. 2006, Nuclear Phys A 777, 424 (astro-ph/0605725)

Ohkubo, T., Umeda, H., Maeda, K., Nomoto, K., Suzuki, T., Tsuruta, S., \& Rees, M. J. 2006, ApJ 645, 1352

Ohkubo, T., Nomoto, K., Umeda, H., Yoshida, N., \& Tsuruta, S. 2008, ApJ submitted

Poelarends, A. J. T., Herwig, F., Langer, N., \& Heger, A. 2008, ApJ 675, 614

Portezies Zwart, S. F., \& van den Heuvel, E. P. J. 2007, Nature 450, 388

Prieto, J. L., et al. 2008, ApJ 681, L9

Smartt, S. J., et al. 2008, MNRAS submitted (arXiv:0809.0403)

Thompson, T. A., et al. 2008, ApJ submitted (arXiv:0809.0510)

Timmes, F. X., \& Woosley, S. E., 1992, ApJ 396, 649

Tominaga, N., Maeda, K., Umeda, H., Nomoto, K., Tanaka, et al. 2007, ApJ 657, L77

Tominaga, N. 2008, ApJ in press (arXiv:0711.4815)

Tumlinson, J. 2006, ApJ 641, 1

Turatto, M., et al. 1998, ApJ 498, L129

Umeda, H. \& Nomoto, K. 2002, ApJ 565, 385

Umeda, H. \& Nomoto, K. 2008, ApJ 673, 1014

Wanajo, S., Nomoto, K., Janka, H.-T., Kitaura, F. S., \& Muller, B. 2008, ApJ submitted

Woosley, S. E. \& Bloom, J. S. 2006, ARA\&A 44, 507

Woosley, S. E., Blinnikov, S., \& Heger, A. 2007, Nature 450, 390

Yoshida, N., Omukai, K., \& Hernquist, L. 2008, Science 321, 669

Zampieri, L., et al. 2003, MNRAS 338, 711 


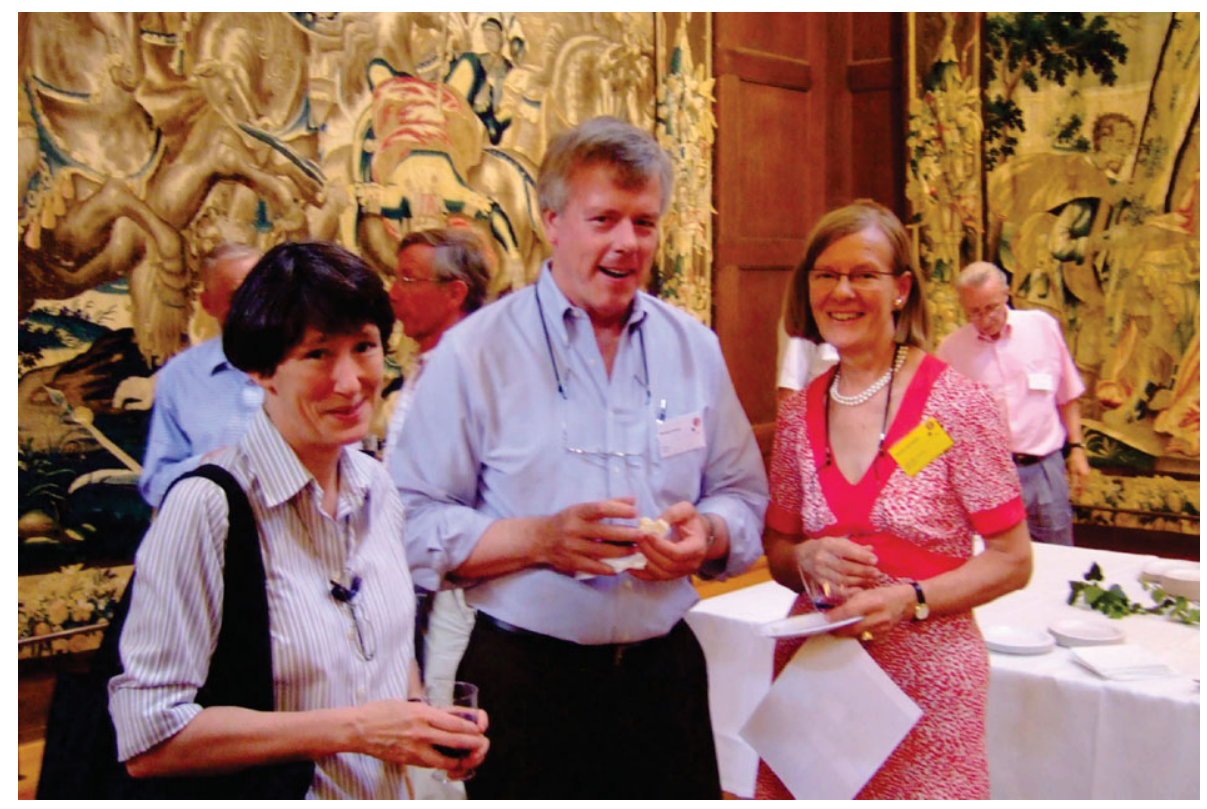

Eileen Friel, Michael Chabin and Birgitta Nordström meet again at the welcome reception.

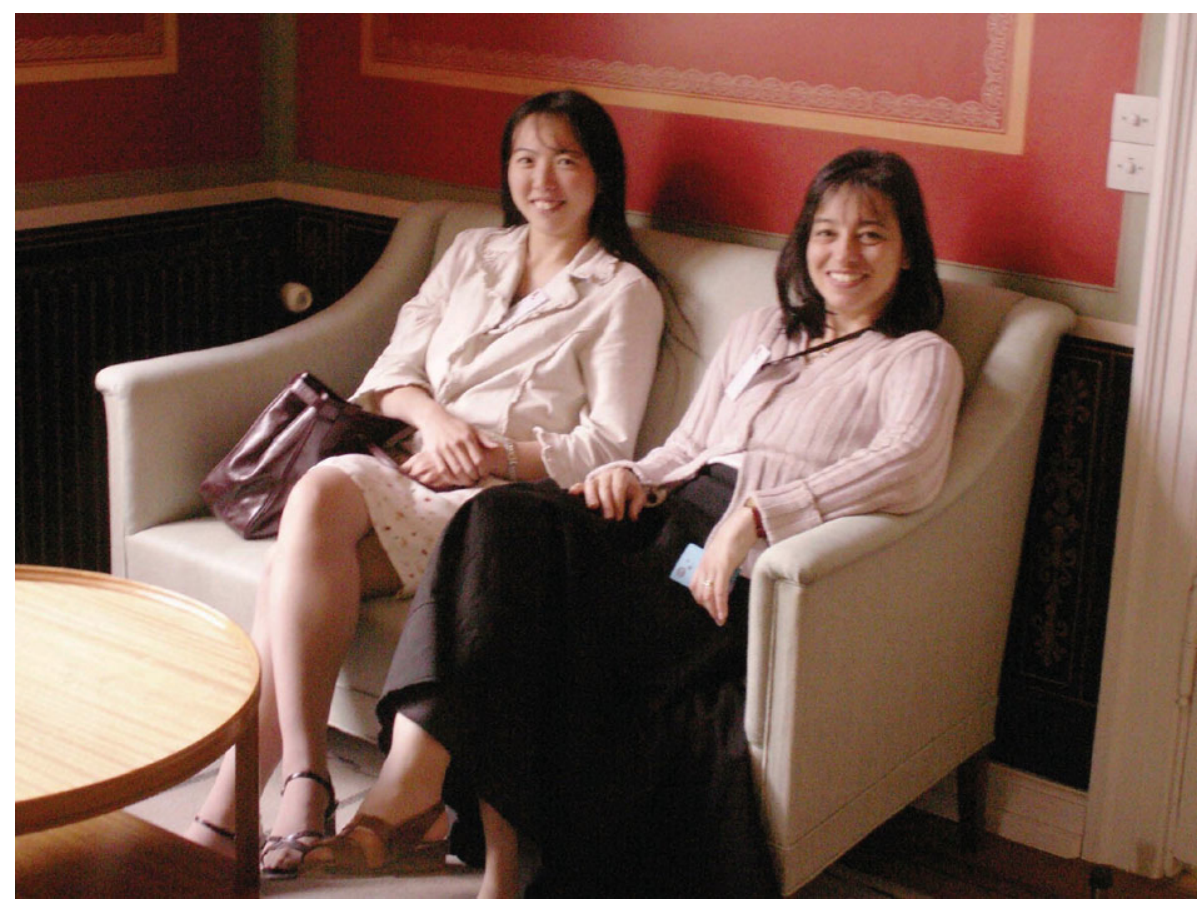

Chiaki Kobayashi and Laura Portinari relaxing offstage at Carlsberg. Photo: Bruce Elmegreen. 